\title{
Deletion of tolA in Salmonella Typhimurium generates an attenuated strain with vaccine potential
}

\begin{abstract}
Correspondence
G. K. Paterson

gkp27@cam.ac.uk
\end{abstract}

Received 20 June 2008

Revised 19 September 2008

Accepted 29 September 2008

\author{
G. K. Paterson, H. Northen, D. B. Cone, C. Willers, S. E. Peters \\ and D. J. Maskell
}

\begin{abstract}
Department of Veterinary Medicine, University of Cambridge, Madingley Road, Cambridge CB3 OES, UK
\end{abstract}

\begin{abstract}
The Gram-negative Tol-Pal system of envelope proteins plays a key role in maintaining outer membrane integrity and contributes to the virulence of several pathogens. We have investigated the role of one of these proteins, TolA, in the biology of Salmonella enterica serovar Typhimurium. Deletion of tolA rendered strain SL1344 more susceptible to killing by bile and human serum. In addition the mutant had impaired membrane integrity and displayed alterations in LPS production. The tolA mutant was highly attenuated in mouse infections via the oral and intravenous routes. Importantly, each phenotype displayed by the mutant was complemented by provision of tolA in trans. The tolA gene therefore contributes to virulence, membrane integrity, LPS production and bile and serum resistance in $S$. enterica serovar Typhimurium SL1344. Finally, immunization with the tolA mutant provided significant protection against subsequent challenge with wild-type SL1344. The Tol-Pal system is therefore a potential target in the development of novel attenuated live vaccines against Salmonella and other Gram-negative pathogens.
\end{abstract}

\section{INTRODUCTION}

Salmonella enterica is a diverse pathogen causing a range of diseases in many hosts, including humans and livestock. As the cause of typhoid fever, S. enterica serovar Typhi is of particular importance to human health, with an estimated annual incidence of 22 million cases and 200000 deaths worldwide (Crump et al., 2004). S. enterica serovar Typhimurium is an important cause of human gastroenteritis and has added significance as a model of human typhoid fever in the mouse.

The Tol-Pal system is well conserved among Gramnegative bacteria (Sturgis, 2001); it consists of (at least) five interacting envelope proteins TolQ, TolR, TolA, TolB and Pal. TolA-Q-R proteins form a complex in the inner membrane while TolB is a periplasmic protein associated with the outer-membrane protein $\mathrm{Pal}$, which itself interacts with peptidoglycan (Henry et al., 2004; Lazzaroni et al., 2002; Lloubes et al., 2001). The system plays numerous roles in the biology of Gram-negative bacteria, including LPS O-antigen surface expression, outer-membrane stability, uptake of filamentous phage DNA, resistance to detergents and virulence (Bowe et al., 1998; Fortney et al., 2000; Gaspar et al., 2000; Heilpern \& Waldor, 2000; Hellman et al., 2002; Lazzaroni et al., 1999; Sun \& Webster, 1987; Vines et al., 2005). For example, tol-pal mutants in the plant pathogen Erwinia chrysanthemi displayed reduced ability to grow in plant tissues, reduced motility, altered morphology and increased susceptibility to various anti- microbial agents (Dubuisson et al., 2005). In the case of $S$. Typhimurium, tolB was identified in a genome-wide screen as a virulence factor in mice contributing to resistance to deoxycholate and serum and bacterial survival in J774 cells (Bowe et al., 1998). The system is also implicated in $S$. Typhimurium bile resistance following the finding that MudJ insertions in the tolQRA cluster result in a bilesensitive phenotype (Prouty et al., 2002). Other than these investigations, few data are available on the function(s) of this system and its individual components in Salmonella, including their role in virulence. A screen of a library of transposon mutants of $S$. Typhimurium in our laboratory identified a tolA mutant as highly attenuated in mice (unpublished observations). tolA was therefore selected for further study and found to be important in virulence, membrane integrity, LPS production and bile and serum resistance in $S$. Typhimurium SL1344. In addition, immunization with the tolA mutant offered protection against subsequent infection with wild-type SL1344.

\section{METHODS}

Generation of a tolA deletion mutant and its complementation. The strains used in this study are shown in Table 1. A $S$. Typhimurium SL1344 tolA mutant was constructed by modification of the ET-cloning procedure (Mo et al., 2006), replacing tolA with the kanamycin-resistance cassette from pUC4Kan (Amersham). PCR was used to amplify the antibiotic-resistance cassette with 5' and 3' $60 \mathrm{bp}$ homology arms complementary to the flanking regions of tolA (see 
Table 1. S. Typhimurium strains used

\begin{tabular}{|lll|}
\hline Name & \multicolumn{1}{c|}{ Description } & Source/reference \\
\hline LB5010 & Restriction-deficient derivative of LT2 & Bullas \& Ryu (1983) \\
SL1344 & Wild-type for this study & Hoiseth \& Stocker (1981) \\
SL1344 tolA & tolA deletion mutant in SL1344 & This study \\
tolA pBR322-tolA ${ }^{+}$ & tolA mutant complemented with tolA cloned into pBR322 & This study \\
tolA pBR322 & tolA mutant carrying empty pBR322 & This study \\
SL3261 & aroA mutant of SL1344; well-characterized live attenuated vaccine strain & Hoiseth \& Stocker (1981) \\
\end{tabular}

Table 2 for primer sequences). S. Typhimurium LB5010 was electroporated with the plasmid pBAD $\lambda$ red and transformants selected on LB agar supplemented with $100 \mu \mathrm{g}$ ampicillin $\mathrm{ml}^{-1}$. A single transformant was grown to an $\mathrm{OD}_{595}$ of 0.25 , then arabinose was added to $0.2 \%(\mathrm{w} / \mathrm{v})$ to induce expression of the phage $\lambda$ genes exo, bet and gam encoded by $\mathrm{pBAD} \lambda$ red and the culture grown to $\mathrm{OD}_{600}$ 0.5. The bacteria were then electroporated with the PCR product generated above (approx. $200 \mathrm{pg}$, prepared using the Qiagen QIAquick PCR purification kit). Mutant colonies were selected on LB agar plates supplemented with $50 \mu \mathrm{g}$ kanamycin $\mathrm{ml}^{-1}$. Allelic replacement of tolA was initially confirmed by PCR and sequencing of the product (Table 2).

The tolA mutation in S. Typhimurium LB5010 was transduced by P22 (Schmieger, 1972) into strain SL1344. Transductants were selected on $50 \mu \mathrm{g}$ kanamycin $\mathrm{ml}^{-1}$ and tolA deletion was confirmed by PCR and sequencing, as described above, and by Southern blotting using the kanamycin cassette as a probe. LPS serotype was confirmed by agglutination with $\mathrm{O} 4$ serotype antisera using $\mathrm{O} 9$ antisera as a negative control (Remel Europe/Oxoid).

For complementation, tolA from S. Typhimurium SL1344 was amplified by PCR (Table 2), purified, digested by HindIII and BamHI, purified again and cloned into HindIII/BamHI-cut pBR322 (New England Biolabs). The resultant plasmid was confirmed by sequencing and designated pBR322-tolA ${ }^{+}$. Empty pBR322 was used as a negative control and both were electroporated into the SL1344 tolA mutant with selection on LB plates containing ampicillin $(100 \mu \mathrm{g}$ $\mathrm{ml}^{-1}$ ). The presence of the correct plasmid was confirmed by PCR using primers flanking the cloning site (Table 2).

Growth in vitro. Overnight cultures were prepared in $10 \mathrm{ml} \mathrm{LB}$ broth by inoculation with three colonies from freshly streaked LB agar plates and incubated with shaking (180 r.p.m.) at $37{ }^{\circ} \mathrm{C}$ for $17.5 \mathrm{~h}$. These cultures were diluted $1: 100000$ into $100 \mathrm{ml}$ fresh, pre-warmed
LB broth and incubated with shaking at $37{ }^{\circ} \mathrm{C}$; samples were taken at the times indicated for $\mathrm{OD}_{595}$ readings and viable counts on $\mathrm{LB}$ agar plates. Exponential generation times were calculated from growth rates between 4 and $6 \mathrm{~h}$.

Motility and morphology. Overnight cultures, prepared as for growth in vitro above, were diluted $1: 10$ in sterile PBS and $1 \mu \mathrm{l}$ was inoculated into the centre of LB agar plates containing $0.4 \%$ agar.

Plates were incubated at $37{ }^{\circ} \mathrm{C}$ for $8 \mathrm{~h}$ and the radius of bacterial movement measured. Each experiment was performed in triplicate. For analyis of morphology, overnight cultures were scanned at $5 \mathrm{kV}$ with a Phillips XL30 FEG scanning electron microscope at 5000$25000 \times$ magnification.

Bile resistance. Stationary-phase cultures were serially diluted and plated on LB agar supplemented with various concentrations of bovine bile (Sigma, catalogue number B3883). Colony-forming units were counted after overnight incubation at $37^{\circ} \mathrm{C}$.

Membrane integrity assay. Membrane integrity was assessed by release of intracellular RNase (Young \& Silver, 1991). Cultures were streaked onto LB agar and overlaid with LB agar supplemented with $1 \%$ type VI Torula yeast RNA (Sigma). After overnight incubation plates were flooded with $0.5 \mathrm{M} \mathrm{HCl}$ for $10 \mathrm{~min}$ to precipitate the remaining RNA. Susceptibility to polymyxin B at $10 \mu \mathrm{g} \mathrm{ml}^{-1}$ (Sigma) was determined as described by Fields et al. (1989).

Serum resistance. Stationary-phase cultures were diluted to $10^{6}$ c.f.u. $\mathrm{ml}^{-1}$ in LB supplemented with $50 \%$ human sera. Samples were incubated at $37{ }^{\circ} \mathrm{C}$ with shaking at 180 r.p.m. and viable counts taken at the times indicated. Untreated and heat-treated sera (45 min at $55{ }^{\circ} \mathrm{C}$ ) were compared to investigate the importance of complement in antimicrobial activity. Mouse sera came from adult female BALB/c mice from Harlan.

Table 2. Primers used in this study

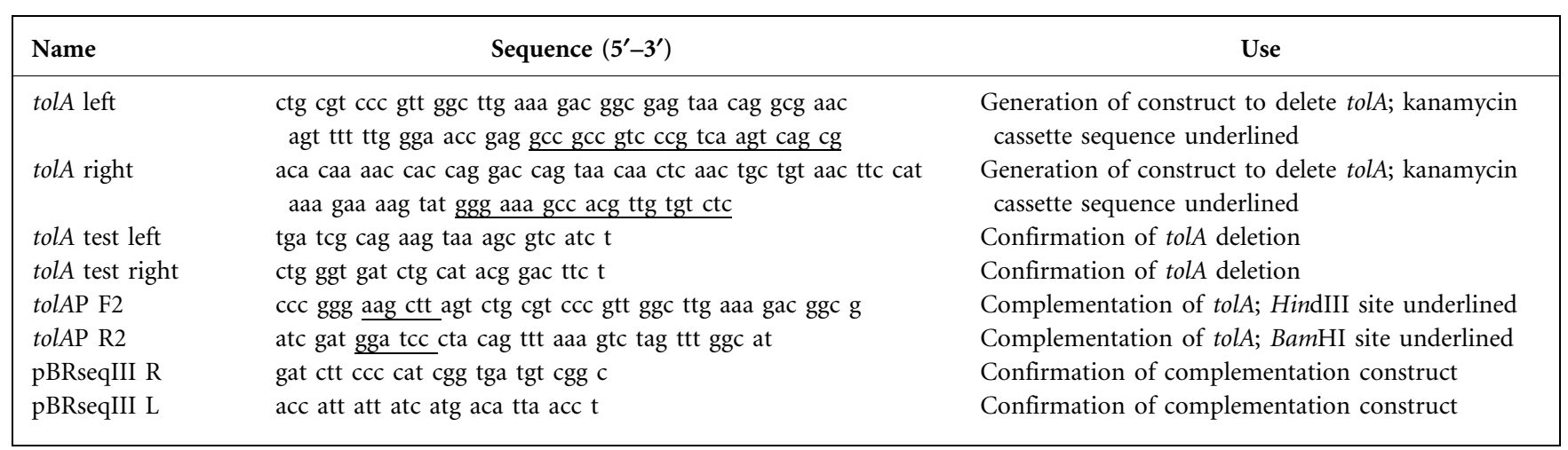


LPS analysis. Stationary-phase cultures were harvested and resuspended in $1 \mathrm{ml}$ PBS and $250 \mu \mathrm{l}$ LPS buffer 1 (0.1875 M Tris/ $\mathrm{HCl} \mathrm{pH} \mathrm{6.8;6 \% ,w/v,} \mathrm{SDS;} 30 \%$, w/v, glycerol) and $20 \mu \mathrm{l}$ of this resuspended cell pellet was boiled for $5 \mathrm{~min}$. Samples were allowed to cool and $70 \mu \mathrm{l}$ LPS buffer $2(0.0625 \mathrm{M}$ Tris/HCl pH 6.8; $0.1 \%$, w/v, SDS; $10 \%, w / v$, glycerol; $0.1 \%, w / v$, bromophenol blue) was added along with proteinase $\mathrm{K}$ to a final concentration of $1.8 \mathrm{mg} \mathrm{ml}^{-1}$. Samples were digested at $55{ }^{\circ} \mathrm{C}$ overnight, separated by electrophoresis using Novex 16\% Tricine gels (Invitrogen) and visualized by silver staining. Equal loading was based on the viable count of the original culture.

Mouse infection models. Bacteria from fresh LB plates were grown statically in $\mathrm{LB}$ broth at $37^{\circ} \mathrm{C}$ overnight. Cultures were then centrifuged at $4300 \mathrm{RCF}$ for $10 \mathrm{~min}$ at $15{ }^{\circ} \mathrm{C}$, resuspended in PBS and adjusted to the required concentration. Female BALB/c mice, 6-8 weeks old (Harlan), were inoculated intravenously with $10^{4}$ c.f.u. or by oral gavage under mild anaesthesia with $10^{8}$ c.f.u.. The dose was confirmed by viable count in LB agar. At the time points indicated, mice were sacrificed and the spleen, liver, Peyer's patches (oral infection only) and mesenteric lymph nodes (oral infection only) were removed into sterile water and homogenized using a Stomacher 80 Lab System (Seward). Bacteria were enumerated by viable counts in LB agar.

For protection studies mice were immunized intravenously with $10^{6}$ c.f.u.. At 13-14 weeks post-infection the primary infection was confirmed to have been cleared from the spleen and liver by viable counting (detection limit 1 c.f.u. per organ, $n=2$ ). Mice were subsequently challenged with wild-type SL1344 by the oral $\left(10^{8}\right.$ c.f.u.) and intravenous $\left(10^{4}\right.$ c.f.u.) routes. Viable counts in the spleen and liver were enumerated as described above. tolA-vaccinated mice were compared to unvaccinated age-matched control mice and mice immunized with the aroA mutant, SL3261.

All work was performed in compliance with UK Home Office regulations under licence.

Statistical analysis. Data were analysed by unpaired Student's $t$-test or one-way ANOVA using GraphPad Version 4, with $P<0.05$ considered as significant.

\section{RESULTS}

\section{Growth in vitro, motility and morphology of SL1344 tolA}

The growth of the tolA mutant in LB broth was compared to wild-type SL1344 as measured by viable count and $\mathrm{OD}_{595}$ (Fig. 1). Growth of the tolA mutant was slightly impaired, as shown by a mean $( \pm$ SEM $)$ exponential generation time of $27.6( \pm 1.5)$ min compared to the wild-type generation time of $23.1( \pm 0.5) \min (P=0.046$, $n=3$ for both).

With regard to motility, there was no significant difference between SL1344 and SL1344 tolA. From one representative experiment the mean $( \pm$ SEM $)$ radius of motility of the wild-type bacteria was $5.4( \pm 0.2) \mathrm{cm}$ while that of the tolA mutant bacteria was $5.1( \pm 0.2) \mathrm{cm}(n=3$ for both $)$.

Overnight cultures when examined by scanning electron microscopy showed no gross difference in morphology between SL1344 and SL1344 tolA (data not shown). Likewise a Gram stain of colonies from LB plates revealed no gross differences (data not shown).

\section{tolA contributes to bile resistance in S. Typhimurium}

MudJ transposon insertions in the orfX-oft1-tolQRA operon led to reduced resistance to bile in $S$. Typhimurium (Prouty et al., 2002). However, none of these insertions were in tolA and so it is unclear if tolA itself contributes to bile resistance. To test directly if tolA does indeed influence bile resistance, cultures were plated onto LB agar supplemented with different concentrations of bovine bile. The tolA mutant was significantly more sensitive to killing by bile compared to the wild-type at bile concentrations of $\geqslant 2.5 \%$ (w/v) (Fig. 2). Confirming that this effect was due to deletion of tolA, this phenotype was complemented by introduction of pBR322tolA ${ }^{+}$but not by empty pBR322 alone (Fig. 2).

\section{S. Typhimurium tolA has impaired membrane integrity}

To test if tolA contributes to the membrane integrity of $S$. Typhimurium the release of intracellular RNase was assessed (Fig. 3a). In this qualitative assay more RNase was released by the tolA mutant than by wild-type SL1344 as shown by the zones of clearance surrounding colonies. Again, the wild-type phenotype was restored in the mutant by introduction of pBR322-tol $A^{+}$but not by the empty vector (Fig. 3a). To test the membrane integrity of the tolA mutant further, strains were exposed to the outermembrane-damaging antimicrobial peptide polymyxin B. Compatible with impaired membrane integrity the tolA
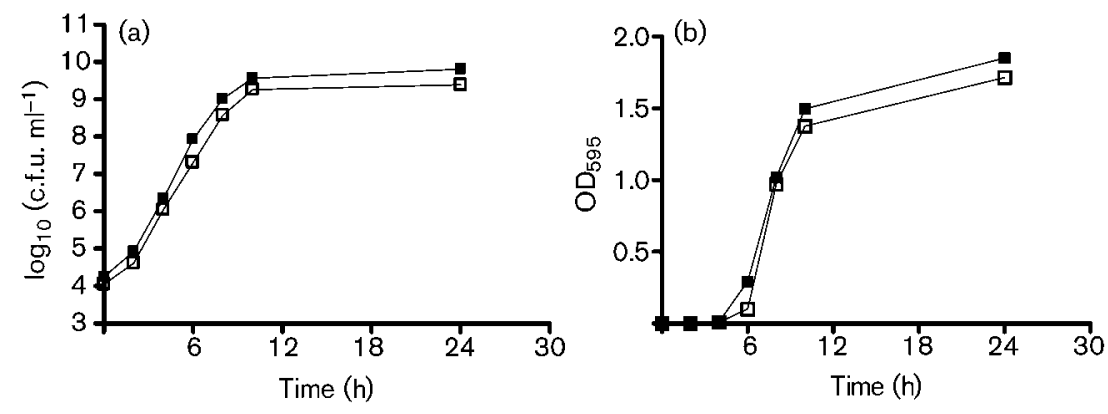

Fig. 1. Growth of SL1344 tolA in LB broth.
Overnight cultures of SL1344 wild-type ( $\mathbf{a})$
and SL1344 tolA ( $\square$ ) were inoculated into
fresh pre-warmed LB and grown with shaking
at $37{ }^{\circ} \mathrm{C}$ at 180 r.p.m. Growth was measured
at the times indicated by viable counts (a) and
$\mathrm{OD}_{595}$ (b). The data are representative of three
experiments giving similar results. 


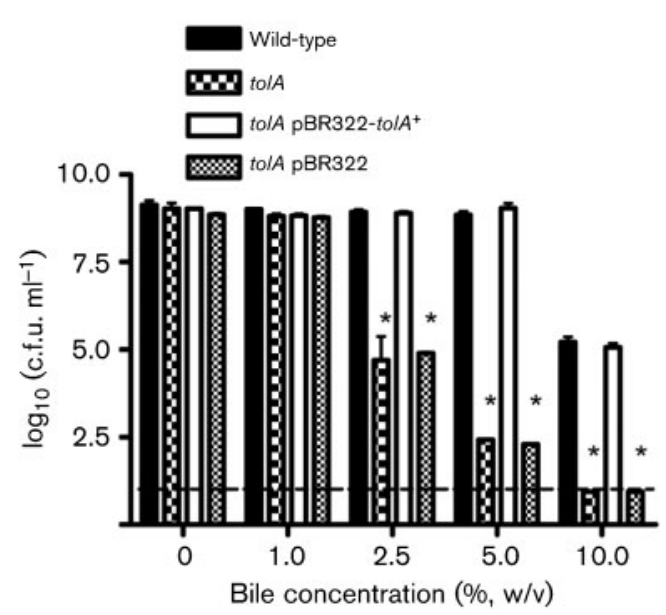

Fig. 2. Bile resistance in S. Typhimurium SL1344 wild-type and its tolA mutant. After overnight growth, LB broth cultures were diluted onto LB agar plates supplemented with bovine bile (Sigma) at different concentrations and the viable counts determined. The data (means \pm SEM) are representative of three experiments each performed in duplicate. ${ }^{\star} P<0.05$ compared to wild-type. The dashed line indicates the limit of detection.

mutant was significantly more susceptible than wild-type SL1344 to killing by polymyxin B (Fig. 3b). Introduction of pBR322-tolA ${ }^{+}$restored a wild-type phenotype to the tolA mutant, while the empty plasmid had no effect (Fig. 3b).

\section{tolA enhances serum resistance in S. Typhimurium}

Wild-type and tolA mutant bacteria were grown in $50 \%$ human serum to investigate if tolA contributes to serum resistance (Fig. 4a). The tolA mutant was significantly more sensitive than the wild-type to human serum and this phenotype was complemented by pBR322-tolA ${ }^{+}$. All strains grew equally well in heat-treated serum, confirming the role of complement in serum killing (Fig. 4b). When human serum was replaced by mouse serum all strains grew equally well, even in untreated serum (data not shown), which is consistent with the fact that $S$. Typhimurium is highly resistant to mouse serum.

\section{tolA contributes to LPS biosynthesis}

Surface expression of Escherichia coli LPS O-antigen was significantly impaired in the absence of tolA (Gaspar et al., 2000; Vines et al., 2005). To visualize the effect of tolA deletion on LPS production in S. Typhimurium, proteinase K-digested bacterial lysates were separated by SDS-PAGE and silver stained (Fig. 5). In the tolA mutant the LPS molecule was slightly altered, with a reduction in the abundance of medium-length LPS polymers (Fig. 5a) and a concomitant increase in low-molecular-mass species most apparent in underdeveloped gels (Fig. 5b), i.e. when gels

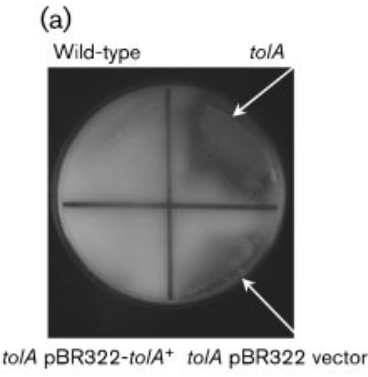

(b)

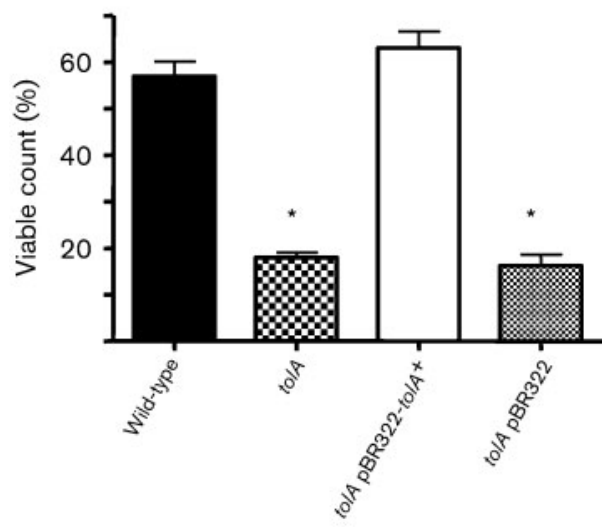

Fig. 3. (a) Membrane leakage in $S$. Typhimurium tolA. Cultures were streaked onto LB agar and overlain with LB agar supplemented with $1 \%$ type VI Torula yeast RNA (Sigma). After overnight incubation plates were flooded with $0.5 \mathrm{M} \mathrm{HCl}$ to precipitate the remaining RNA. Release of RNase is shown by a zone of clearance in the precipitation, indicated by white arrows. (b) Susceptibility to polymyxin B killing. Strains were grown in the presence of polymyxin $B\left(10 \mu \mathrm{g} \mathrm{m}^{-1}\right)$ and viable counts after $1 \mathrm{~h}$ expressed as a percentage of the count in the absence of polymyxin $B$. The data are representative of two experiments giving similar results, each performed in duplicate. ${ }^{*} P<0.05$ compared to wild-type.

were developed only long enough for the more abundant species to be apparent. Again, this phenotype was reversed by introduction of pBR322-tolA ${ }^{+}$but not by the empty vector (Fig. 5).

\section{tolA contributes to $S$. Typhimurium virulence in the mouse}

The multiple effects of tolA deletion on the phenotype of SL1344 suggested that tolA might contribute to the virulence of $S$. Typhimurium. To test this, BALB/c mice were infected intravenously with $10^{4}$ c.f.u. of wild-type or tolA mutant bacteria and bacterial loads in the spleen and liver enumerated over time (Fig. 6). In both organs the bacterial count was significantly lower for mice infected with the tolA mutant versus those infected with wild-type SL1344. Unlike wild-type bacteria, the tolA mutant showed no net growth between days 1 and 3 post-infection and at 
(a)

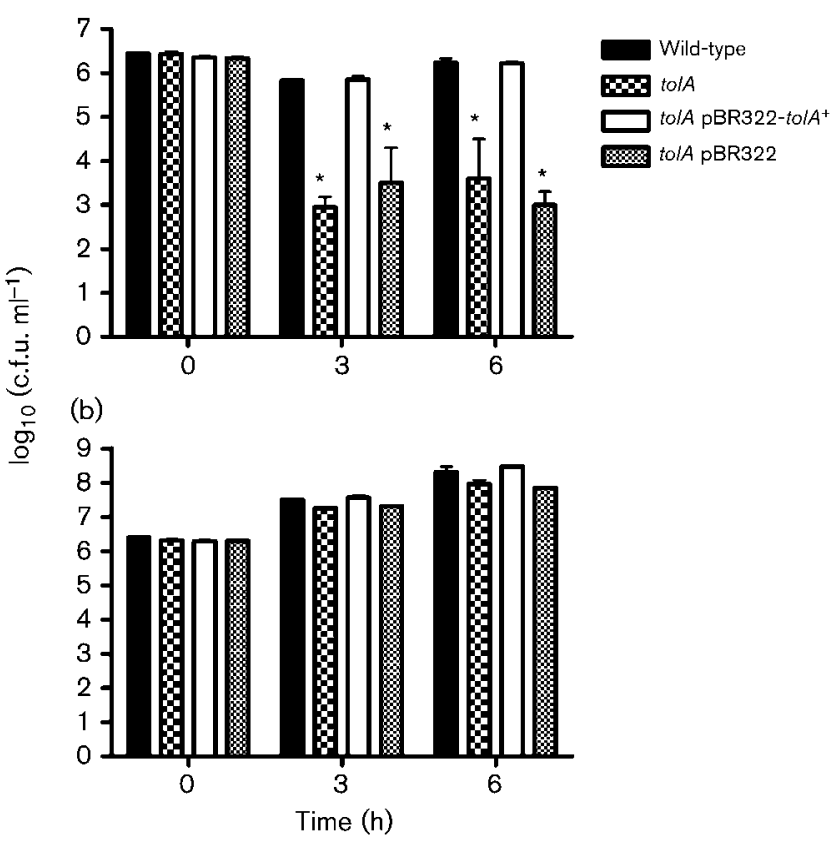

Fig. 4. Serum resistance of $S$. Typhimurium SL1344 wild-type and its tolA mutant. (a) Strains were incubated in $50 \%$ fresh human serum diluted in LB broth and viable counts taken at the times indicated. (b) Heat treatment to abolish complement activity was performed at $55{ }^{\circ} \mathrm{C}$ for $45 \mathrm{~min}$. The data are means \pm SEM of a representative experiment from three experiments each using different donor serum, each performed in duplicate and giving similar results. ${ }^{*} P<0.05$ compared to wild-type.

day 3 post-infection wild-type counts were approximately $10^{4}$-fold greater than the mutant counts. The mutant phenotype was complemented by pBR322-tolA ${ }^{+}$while the empty pBR322 vector had no effect (Fig. 6).

The contribution of tolA to virulence was further assessed following oral inoculation, the natural route of infection. Four days after oral infection, bacterial loads in the spleen, liver, Peyer's patches and mesenteric lymph nodes were enumerated (Fig. 7). In all four organs bacterial counts were significantly lower in the mice infected with the tolA mutant compared to those infected with wild-type bacteria. Just as with all the other tolA mutant phenotypes described here the defect was restored by pBR322-tolA ${ }^{+}$but not by the empty pBR322 vector.

\section{Immunization with SL1344 to/A protects against subsequent wild-type challenge}

Following immunization with the tolA mutant, mice were rechallenged with wild-type SL1344 via the oral and intravenous routes (Fig. 8). In comparison with unimmunized age-matched control mice, the mice immunized with the tolA mutant had significantly reduced

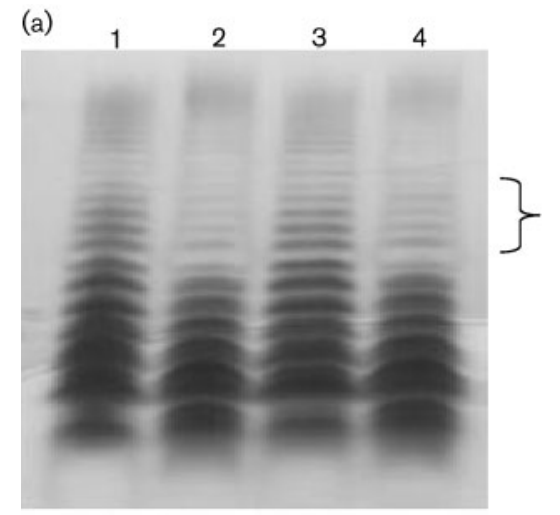

(b)

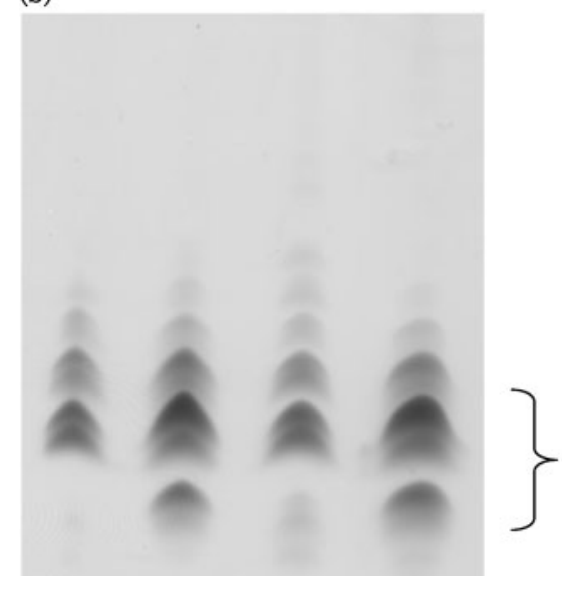

Fig. 5. Analysis of LPS from S. Typhimurium SL1344 wild-type and its tolA mutant. Proteinase $\mathrm{K}$-digested cell lysates from approximately $5 \times 10^{7}$ c.f.u. were separated by SDS-PAGE and silver stained. (a) Fully developed (no more bands likely to became apparent) and (b) underdeveloped (only the most abundant bands visible) gels show subtle alterations in LPS between wild-type and SL1344 tolA, indicated by brackets. Lanes: 1, SL1344; 2, SL1344 tolA; 3, SL1344 tolA pBR322-tolA ${ }^{+} ; 4$, SL1344 tolA pBR322.

bacterial counts in the spleen and liver. The tolA mutant is therefore immunogenic and protective against subsequent challenge. However, immunization with the tolA mutant did not reduce bacterial counts as effectively as immunization with the aroA mutant SL3261 (Fig. 8).

\section{DISCUSSION}

The tol-pal system is relatively uncharacterized in $S$. Typhimurium but is likely to play key roles in the biology of this important pathogen given the functions of this complex in other Gram-negative bacteria. The tolA gene was selected for study because it was identified by us as a candidate gene contributing to bacterial virulence in a large-scale transposon mutagenesis screen in mice (unpublished observations). Therefore, to investigate the roles of the Gram-negative envelope protein TolA in the biology of 

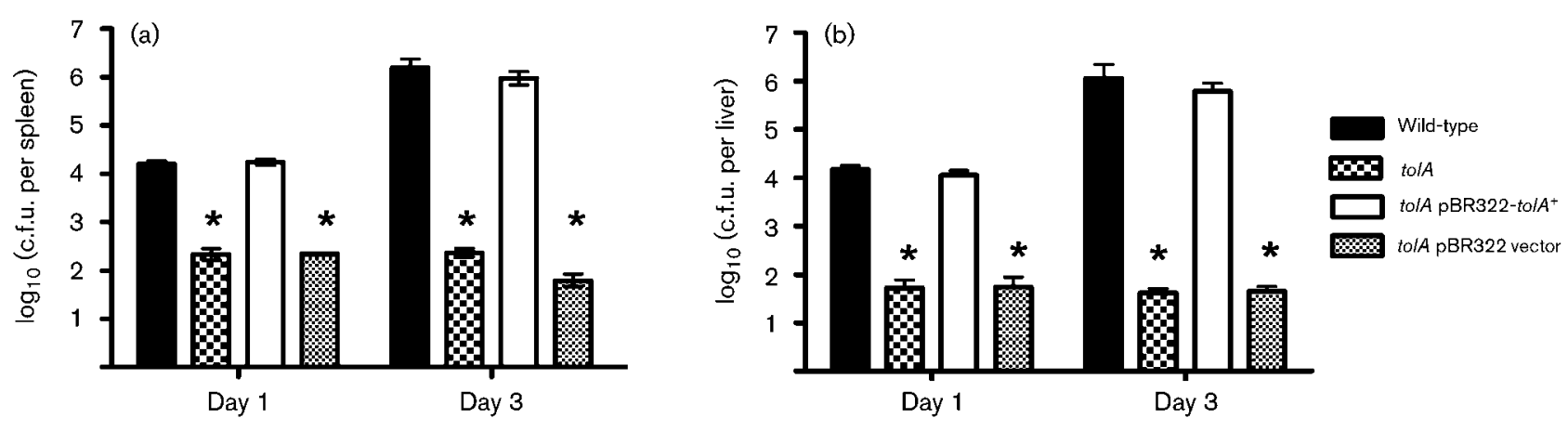

Fig. 6. Bacterial counts in the spleen (a) and liver (b) following intravenous infection with $10^{4}$ c.f.u. S. Typhimurium SL1344 wild-type and its tolA mutant. The data are means \pm SEM $(n=6-7)$ pooled from two experiments giving similar results. ${ }^{\star} P<0.05$ compared to wild-type.

Salmonella we generated a defined tolA deletion in $S$. Typhimurium SL1344. Various tol-pal gene deletions in E. chrysanthemi, including a tolA mutant, displayed altered morphology and decreased motility (Dubuisson et al., 2005). Neither phenotype was shared by our tolA mutant in $S$. Typhimurium, suggesting species-specific effects for the tol-pal system. Previously transposon insertions in the orfX-oft1-tolQRA operon reduced resistance to bile in $S$. Typhimurium; however, none of these was in tolA (Prouty et al., 2002). Our S. Typhimurium tolA mutant was more susceptible to bile than its wild-type counterpart; this appears to be the first demonstration of a role in bile resistance for the tolA gene. Resistance to bile is an important trait for enteric bacteria and the Tol-Pal system is clearly a vital component of this resistance in $S$. Typhimurium. This bile-sensitive phenotype may have contributed to the attenuation shown by the tolA mutant via the oral, but not the intravenous, route of infection. (a)

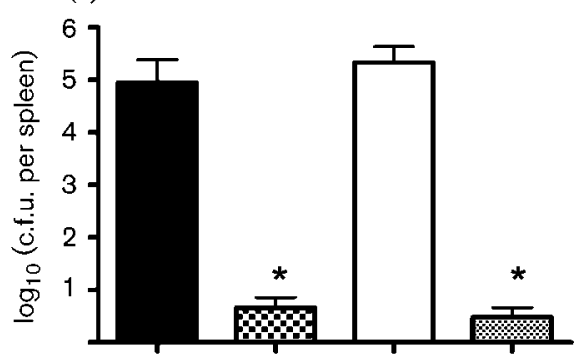

(b)

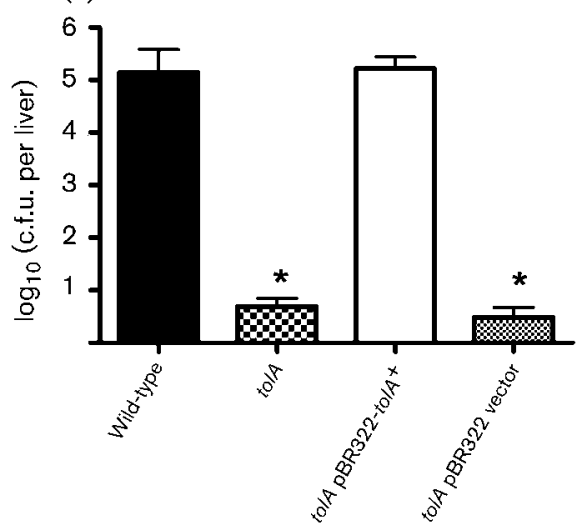

(c)

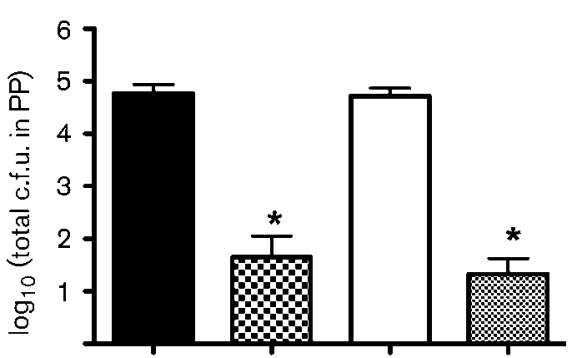

(d)

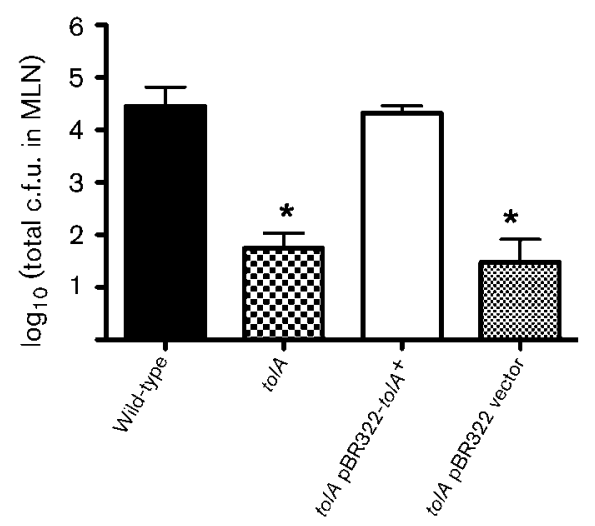

Fig. 7. Bacterial counts in the spleen (a), liver (b), Peyer's patches (PP, c) and mesenteric lymph nodes (MLN, d) 4 days after oral infection with $10^{8}$ c.f.u. S. Typhimurium SL1344 wild-type and its to/A mutant. The data are means \pm SEM $(n=9-10)$ pooled from two experiments giving similar results. ${ }^{*} P<0.05$ compared to wild-type. 

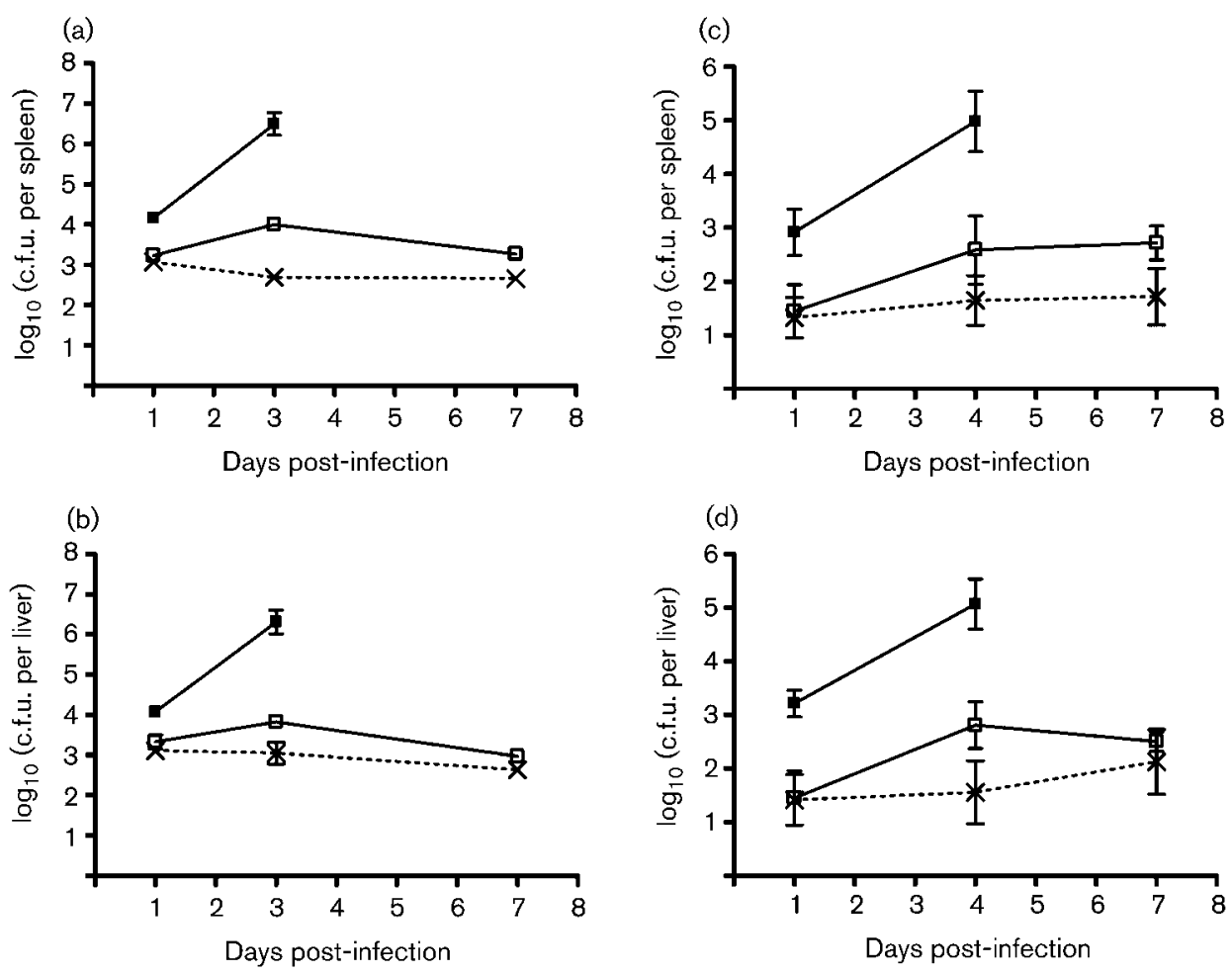

Fig. 8. Immunization with SL1344 tolA protects against subsequent wild-type infection. Mice were immunized intravenously with $10^{6}$ c.f.u. SL1344 tolA ( $\square$ ) or SL3261 (x). Following clearance of the immunizing strain, mice, including un-immunized age-matched controls $(\boldsymbol{\square})$, were subsequently challenged with wild-type SL1344 via the intravenous (10 ${ }^{4}$ c.f.u.) $(a, b)$ and oral $\left(10^{8}\right.$ c.f.u.) (c, d) routes. Bacterial counts in the spleen $(a, c)$ and liver $(b, d)$ were determined. Immunization with SL1344tolA and SL3261 offered significant protection compared to un-immunized controls $(P<0.05)$. Data (means \pm SEM, $n=3-4)$ are representative of two experiments giving similar results. Data for un-immunized mice at day 7 were not collected for ethical reasons, as the mice would not be expected to survive until this time point.

SL1344 tolA displayed reduced membrane integrity as demonstrated by release of intracellular RNase. This phenotype is shared with tol-pal mutants in E. coli and was seen following tolB deletion in $S$. Typhimurium (Bowe et al., 1998). Bowe et al. (1998) suggested that this membrane phenotype could render tolB mutants more susceptible to harsh conditions in the host and thus contribute to reduced virulence via both the oral and intravenous routes. This is likely to apply to tolA mutants too. In support of this we found that deletion of tolA increased susceptibility to the antimicrobial peptide polymyxin B.

The tolA mutant was also more susceptible to killing by human serum. This effect was lost upon heating, showing that complement is required for this killing. No microbicidal effect was seen when using mouse serum; thus the complement-mediated serum killing of $S$. Typhimurium SL1344 depends on the source of serum. This agrees with previous data showing that mouse serum had little killing activity (Jones et al., 1997; Nagy et al., 2004; Ohno et al., 1995). Indeed, even deep rough Salmonella mutants are not susceptible to killing by mouse serum (Ohno et al., 1995).
It therefore seems that increased killing by serum does not contribute to the attenuation of the tolA mutant in the mouse.

In E. coli, tolA mutation reduces $\mathrm{O}$-antigen surface expression through defective Wzy polymerization (Gaspar et al., 2000; Vines et al., 2005). We show here that $S$. Typhimurium tolA also displays a difference in LPS production but this is much more subtle than in E. coli, with only a small reduction in medium-length polymers and an associated increase in short-chain species. The latter effect was only apparent on underdeveloped gels, probably due to the abundance of these species causing rapid saturation of signal. LPS protects Salmonella against environmental assaults such as bile and complement and contributes to virulence (Miller et al., 1989; Murray et al., 2003, 2006; Ohno et al., 1995; Prouty et al., 2002; Thomsen et al., 2003). Therefore, although subtle, this alteration of LPS in tolA may contribute to the other phenotypes described here. This may especially be the case with regard to resistance to human serum, which is associated with medium-length $\mathrm{O}$-antigen containing between 4 and 15 repeat units (Murray et al., 2006). 
The SL344 tolA mutant was highly attenuated in mice following intravenous and oral inoculation, most likely as a result of the phenotypes we describe relating to impaired membrane integrity and altered LPS production. Attenuation was assessed based on viable counts in the spleen and liver following intravenous infection, and in the spleen, liver, Peyer's patches and mesenteric lymph nodes following oral inoculation. In each case the tolA mutant counts were significantly lower than those seen for wildtype SL1344, demonstrating the requirement for tolA for full virulence in these models. Work is now under way to compare the different Tol-Pal system components for the phenotypes described here. For example, will all the Salmonella tol-pal gene products be equally important for membrane integrity, LPS biosynthesis and virulence? Data from E. chrysanthemi suggests that they have differing influences (Dubuisson et al., 2005).

Finally, immunization with SL1344 tolA offered significant protection against subsequent SL1344 wild-type challenge. This is believed to be the first demonstration that mutation of the tol-pal system has potential utility in the development of live attenuated vaccine strains in Salmonella and other Gram-negative pathogens.

\section{ACKNOWLEDGEMENTS}

This work was supported by a BBSRC Applied Genomics Link Grant and a BBSRC Project Grant. Electron microscopy was carried out at the Multi-Imaging Centre, University of Cambridge, by Ewelina Michalowska and Jeremy Skepper.

\section{REFERENCES}

Bowe, F., Lipps, C. J., Tsolis, R. M., Groisman, E., Heffron, F. \& Kusters, J. G. (1998). At least four percent of the Salmonella typhimurium genome is required for fatal infection of mice. Infect Immun 66, 3372-3377.

Bullas, L. R. \& Ryu, J. I. (1983). Salmonella typhimurium LT2 strains which are $\mathrm{r}^{-} \mathrm{m}^{+}$for all three chromosomally located systems of DNA restriction and modification. J Bacteriol 156, 471-474.

Crump, J. A., Luby, S. P. \& Mintz, E. D. (2004). The global burden of typhoid fever. Bull World Health Organ 82, 346-353.

Dubuisson, J. F., Vianney, A., Hugouvieux-Cotte-Pattat, N. \& Lazzaroni, J. C. (2005). Tol-Pal proteins are critical cell envelope components of Erwinia chrysanthemi affecting cell morphology and virulence. Microbiology 151, 3337-3347.

Fields, P. I., Groisman, E. A. \& Heffron, F. (1989). A Salmonella locus that controls resistance to microbicidal proteins from phagocytic cells. Science 243, 1059-1062.

Fortney, K. R., Young, R. S., Bauer, M. E., Katz, B. P., Hood, A. F., Munson, R. S., Jr \& Spinola, S. M. (2000). Expression of peptidoglycanassociated lipoprotein is required for virulence in the human model of Haemophilus ducreyi infection. Infect Immun 68, 6441-6448.

Gaspar, J. A., Thomas, J. A., Marolda, C. L. \& Valvano, M. A. (2000). Surface expression of O-specific lipopolysaccharide in Escherichia coli requires the function of the TolA protein. Mol Microbiol 38, 262-275.

Heilpern, A. J. \& Waldor, M. K. (2000). CTX $\phi$ infection of Vibrio cholerae requires the tolQRA gene products. J Bacteriol 182, 1739-1747.
Hellman, J., Roberts, J. D., Jr, Tehan, M. M., Allaire, J. E. \& Warren, H. S. (2002). Bacterial peptidoglycan-associated lipoprotein is released into the bloodstream in gram-negative sepsis and causes inflammation and death in mice. J Biol Chem 277, 14274-14280.

Henry, T., Pommier, S., Journet, L., Bernadac, A., Gorvel, J.-P. \& Lloubès, R. (2004). Improved methods for producing outer membrane vesicles in Gram-negative bacteria. Res Microbiol 155, 437-446.

Hoiseth, S. K. \& Stocker, B. A. (1981). Aromatic-dependent Salmonella typhimurium are non-virulent and effective as live vaccines. Nature 291, 238-239.

Jones, B. D., Nichols, W. A., Gibson, B. W., Sunshine, M. G. \& Apicella, M. A. (1997). Study of the role of the htrB gene in Salmonella typhimurium virulence. Infect Immun 65, 4778-4783.

Lazzaroni, J. C., Germon, P., Ray, M. C. \& Vianney, A. (1999). The Tol proteins of Escherichia coli and their involvement in the uptake of biomolecules and outer membrane stability. FEMS Microbiol Lett 177, 191-197.

Lazzaroni, J.-C., Dubuisson, J.-F. \& Vianney, A. (2002). The Tol proteins of Escherichia coli and their involvement in the translocation of group A colicins. Biochimie 84, 391-397.

Lloubes, R., Cascales, E., Walburger, A., Bouveret, E., Lazdunski, C., Bernadac, A. \& Journet, L. (2001). The Tol-Pal proteins of the Escherichia coli cell envelope: an energized system required for outer membrane integrity? Res Microbiol 152, 523-529.

Miller, I., Maskell, D., Hormaeche, C., Johnson, K., Pickard, D. \& Dougan, G. (1989). Isolation of orally attenuated Salmonella typhimurium following TnphoA mutagenesis. Infect Immun 57, 2758-2763.

Mo, E., Peters, S. E., Willers, C., Maskell, D. J. \& Charles, I. G. (2006). Single, double and triple mutants of Salmonella enterica serovar Typhimurium $\operatorname{deg} P$ (htrA), $\operatorname{deg} Q(h h o A)$ and $\operatorname{deg} S$ (hhoB) have diverse phenotypes on exposure to elevated temperature and their growth in vivo is attenuated to different extents. Microb Pathog 41, 174-182.

Murray, G. L., Attridge, S. R. \& Morona, R. (2003). Regulation of Salmonella typhimurium lipopolysaccharide $\mathrm{O}$ antigen chain length is required for virulence; identification of FepE as a second Wzz. Mol Microbiol 47, 1395-1406.

Murray, G. L., Attridge, S. R. \& Morona, R. (2006). Altering the length of the lipopolysaccharide $\mathrm{O}$ antigen has an impact on the interaction of Salmonella enterica serovar Typhimurium with macrophages and complement. J Bacteriol 188, 2735-2739.

Nagy, G., Dobrindt, U., Hacker, J. \& Emody, L. (2004). Oral immunization with an $r f a H$ mutant elicits protection against salmonellosis in mice. Infect Immun 72, 4297-4301.

Ohno, A., Isii, Y., Tateda, K., Matumoto, T., Miyazaki, S., Yokota, S. \& Yamaguchi, K. (1995). Role of LPS length in clearance rate of bacteria from the bloodstream in mice. Microbiology 141, 2749-2756.

Prouty, A. M., Van Velkinburgh, J. C. \& Gunn, J. S. (2002). Salmonella enterica serovar Typhimurium resistance to bile: identification and characterization of the tolQRA cluster. J Bacteriol 184, 12701276.

Schmieger, H. (1972). Phage P22-mutants with increased or decreased transduction abilities. Mol Gen Genet 119, 75-88.

Sturgis, J. N. (2001). Organisation and evolution of the tol-pal gene cluster. J Mol Microbiol Biotechnol 3, 113-122.

Sun, T. P. \& Webster, R. E. (1987). Nucleotide sequence of a gene cluster involved in entry of E colicins and single-stranded DNA of infecting filamentous bacteriophages into Escherichia coli. J Bacteriol 169, 2667-2674. 
Thomsen, L. E., Chadfield, M. S., Bispham, J., Wallis, T. S., Olsen, J. E. \& Ingmer, H. (2003). Reduced amounts of LPS affect both stress tolerance and virulence of Salmonella enterica serovar Dublin. FEMS Microbiol Lett 228, 225-231.

Vines, E. D., Marolda, C. L., Balachandran, A. \& Valvano, M. A. (2005), Defective O-antigen polymerization in tolA and pal mutants of
Escherichia coli in response to extracytoplasmic stress. J Bacteriol 187, 3359-3368.

Young, K. \& Silver, L. L. (1991). Leakage of periplasmic enzymes from envA1 strains of Escherichia coli. J Bacteriol 173, 3609-3614.

Edited by: D. L. Gally 